University of Nebraska - Lincoln

DigitalCommons@University of Nebraska - Lincoln

$1-25-2005$

\title{
Atomic force microscopy-based experimental setup for studying domain switching dynamics in ferroelectric capacitors
}

\author{
C. DeHoff \\ North Carolina State University, North Carolina
}

B. J. Rodriguez

North Carolina State University, Campus Box 7920, Raleigh, North Carolina, brian.rodriguez@ucd.ie

A. I. Kingon

North Carolina State University, angus_kingon@brown.edu

R. J. Nemanich

North Carolina State University, Campus Box 7920, Raleigh, North Carolina

Alexei Gruverman

University of Nebraska-Lincoln, agruverman2@unl.edu

See next page for additional authors

Follow this and additional works at: https://digitalcommons.unl.edu/physicsgruverman

Part of the Physics Commons

DeHoff, C.; Rodriguez, B. J.; Kingon, A. I.; Nemanich, R. J.; Gruverman, Alexei; and Cross, J. S., "Atomic force microscopy-based experimental setup for studying domain switching dynamics in ferroelectric capacitors" (2005). Alexei Gruverman Publications. 38.

https://digitalcommons.unl.edu/physicsgruverman/38

This Article is brought to you for free and open access by the Research Papers in Physics and Astronomy at DigitalCommons@University of Nebraska - Lincoln. It has been accepted for inclusion in Alexei Gruverman Publications by an authorized administrator of DigitalCommons@University of Nebraska - Lincoln. 


\section{Authors}

C. DeHoff, B. J. Rodriguez, A. I. Kingon, R. J. Nemanich, Alexei Gruverman, and J. S. Cross 


\title{
Atomic force microscopy-based experimental setup for studying domain switching dynamics in ferroelectric capacitors
}

\author{
C. Dehoff, ${ }^{\text {a) }}$ B. J. Rodriguez, A. I. Kingon, R. J. Nemanich, and A. Gruverman ${ }^{\text {b) }}$ \\ Departments of Materials Science and Engineering and Physics, North Carolina State University, Campus \\ Box 7920, Raleigh, North Carolina 27695-7919 \\ J. S. Cross \\ Fujitsu Laboratories Ltd., Atsugi, Japan
}

(Received 7 September 2004; accepted 29 November 2004; published online 25 January 2005)

This article describes an experimental setup for combined measurements of domain switching dynamics and switching currents in micrometer scale ferroelectric capacitors. The setup is based on a commercial atomic force microscope (AFM) that is equipped with a piezoresponse mode for domain imaging and with a wide bandwidth current amplifier for switching current recording. The setup allows combined domain/current measurements in capacitors as small as $1 \mu \mathrm{m}^{2}$ with switching times resolved down to $10 \mathrm{~ns}$. The incorporation of switching current measurement capability into piezoresponse AFM makes detailed analysis of switching behavior in ferroelectric memory devices possible. (C) 2005 American Institute of Physics. [DOI: 10.1063/1.1850652]

\section{INTRODUCTION}

Recently, nonvolatile ferroelectric random access memories (NVFRAMs) began attracting significant attention due to a number of advantageous properties that make them highly competitive with other nonvolatile memories, such as flash devices. Low power consumption and long storage times are important for applications in mobile devices, which are generally limited by their battery power. Furthermore, NVFRAMs provide faster access times and higher endurance than conventional memories. ${ }^{1}$ However, for NVFRAM to become a superior memory technology, ferroelectric capacitors of micro- and submicrometer dimensions need to be developed and their electrical parameters well characterized.

One of the most important questions is the relation between capacitor scaling and the ferroelectric switching behavior of the capacitors. There are several well-documented studies of quasistatic and pulse switching behavior in micrometer size capacitors. ${ }^{2-5}$ However, in order to employ a ferroelectric capacitor for NVFRAM applications, there is also a critical need to understand the domain dynamics during ferroelectric switching. ${ }^{2}$

Switching current measurements can be used to extract information about the switched charge, switching time, and activation field. From the value of the activation field, the absolute minimum voltage necessary to switch the domains can be calculated for a film of known thickness. The voltagedependent duration of the entire switching process, which determines the minimum time for a read-out or write process in the FRAM device, can be determined by monitoring the

\footnotetext{
a) On leave from Aachen University; electronic mail: Carsten.Dehoff@rwthaachen.de

b) Author to whom correspondence should be addressed; electronic mail: Alexei_Gruverman@ncsu.edu
}

switching current. Recording the domain dynamics during polarization reversal can provide additional insight on spatial variations in the switching parameters.

Measurements of the switching currents in individual micron and submicron ferroelectric capacitors became possible after the introduction of the atomic force microscopy (AFM), which provided the capability of connecting a conductive probe precisely to the top electrode of the capacitor. Recently, Prasertchoung et al. reported measurements of dynamic switching currents in capacitors down to $0.19 \mu \mathrm{m}^{2}{ }^{6}$ In addition, the piezoresponse mode of AFM, called piezoresponse force microscopy (PFM), allowed visualization of the domain structure in thin film capacitors. ${ }^{7,8} \mathrm{PFM}$ is also widely used to address degradation effects in ferroelectrics, such as imprint and fatigue., ${ }^{9,10}$

Most of the previous PFM-related papers focused on investigation of static domain configurations before and after application of a switching voltage. One attempt to investigate domain growth dynamics during switching in ferroelectric capacitors was undertaken by Hong et al. ${ }^{11}$ who observed nucleation of domains and their subsequent sidewise expansion by using an incrementally increasing dc bias. In this article, we describe a setup that allows the measurement of switching current along with PFM imaging of domain dynamics during switching. Here, to induce polarization reversal, we employ a sequence of voltage pulses with incrementally increasing duration and fixed amplitude above the threshold voltage. This approach more closely emulates the measurement procedure used for testing NVFRAM devices. So, information on both switching current and domain growth dynamics can be obtained. Furthermore, the influence of backswitching effects on the switching current can be investigated. Our setup allows one to combine PFM imaging and current measurement without averaging for cells down to $3 \times 3 \mu \mathrm{m}^{2}$ (with averaging-down to $1 \times 1.5 \mu \mathrm{m}^{2}$ ) and can address how real switching dynamics in ferroelectric capacitors relates to the various switching models. ${ }^{12-14}$ 
(a)
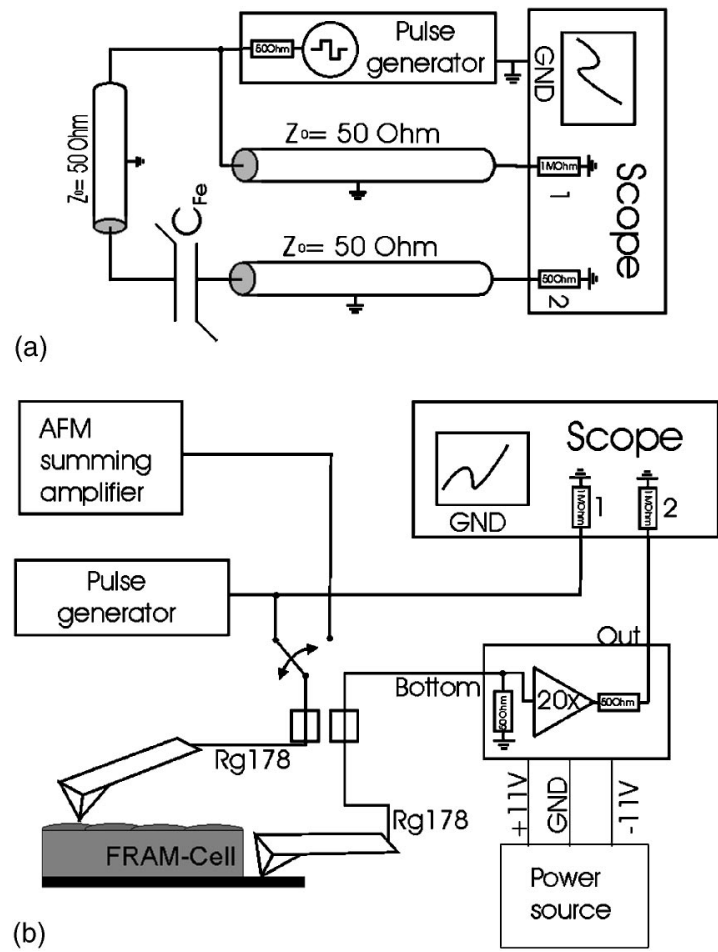

FIG. 1. Circuit scheme of measurement circuit: (a) Simplified, including cable impedance. (b) Complete, without cable impedance but including AFM connection and an amplifier.

\section{EXPERIMENTAL SETUP}

Samples used in this study were 200 -nm thick capacitors of tetragonal lead zirconate titanate (PZT) sputtered onto a Pt bottom electrode. Reactive ion etching has been used to fabricate capacitors with 50-nm thick $\mathrm{IrO}_{2}$ top electrodes on the PZT surface. Further details on sample composition and preparation can be found in Ref. 2. In this article, 3 $\times 3 \mu \mathrm{m}^{2}$ and $5 \times 5 \mu \mathrm{m}^{2}$ PZT capacitors have been studied.

Details of the PFM imaging method are described elsewhere. ${ }^{15,16}$ PFM employs a voltage signal at a certain frequency $f$, which initiates a piezoelectric motion of the ferroelectric sample. With a lock-in amplifier the piezoelectric based motion can be filtered and quantified. The linear coupling between the piezoelectric and polarization constants allows interpretation of the map of the piezoelectric response as a polarization distribution map. The advantage of this method is that the domain pattern can be detected even through the top electrode of the capacitor.

A simplified circuit diagram of the resistive current sensing circuit $^{17}$ is shown in Fig. 1(a). The experimental setup consists of a commercial AFM (Autoprobe M5 Park Scientific Instruments), a Fluke PM5138 pulse generator, a TEK 3014 digital phosphor oscilloscope, and a Texas Instruments THS4021CD evaluation module board.

The complete setup, including the connection to the AFM is shown in Fig. 1(b). The $50 \Omega$ oscilloscope impedance is used as both termination impedance and measurement shunt. It is important to terminate the system in a proper way in order to avoid signal reflection back to the (FRAM) cell. In case of small capacitors $\left(3 \times 3 \mu \mathrm{m}^{2}\right.$ and smaller) there may be an amplifier installed between bottom

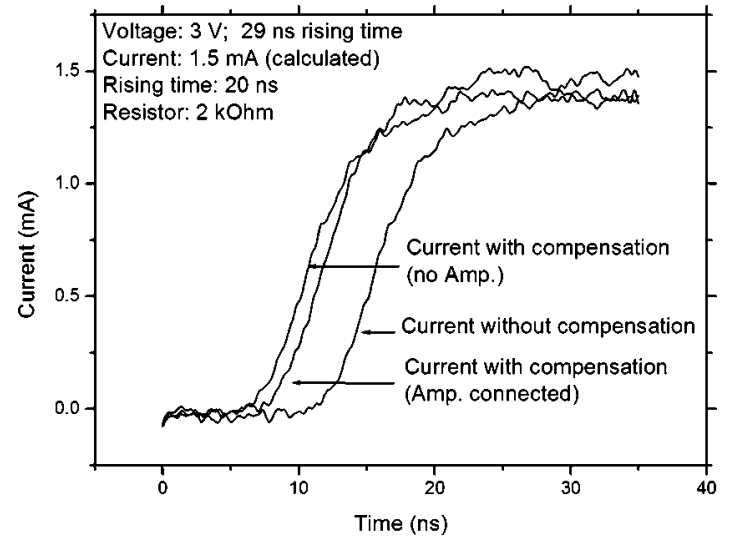

FIG. 2. Calibration results and rising time comparison for switching current measurement system for a $2 \mathrm{k} \Omega$ resistor showing signal delays.

contact and the input of the scope. In this case, the scope termination is set to $10^{6} \Omega$ because the termination is included in the amplifier output.

Since the cable lengths are not the same, this setup causes the signal delay between the reference signal and the measured current signal to differ. Furthermore, the amplifier causes a signal delay time while processing the signal. Connecting a resistor of known resistance with the setup resulted in a delay time of $5 \mathrm{~ns}$. This system related delay time was software compensated. When connected to the capacitor, the rising time increases from $10 \mathrm{~ns}$ (that is the generator is capable to achieve) to $18-30 \mathrm{~ns}$. The current response with a connected $2 \mathrm{k} \Omega$ resistor is shown in Fig. 2. The corresponding voltage pulse, which was applied during this measurement, is shown in Fig. 3. The output channel of the summing amplifier is directly connected with the AFM tip, using a $50 \Omega$ BNC cable.

The optional amplifier allows transient currents as low as $10 \mu \mathrm{A}$ to be measured. The minimum measurable current is not determined by the gain of the amplifier, but rather by noise, which is picked up from the unshielded connection of the back electrode (approx. 3-4 $\mathrm{mm}^{2}$ ) and the missing ground connection at the tip side of the coax cable that is connected to the bottom electrode. Furthermore, the AFM laser electronics is working with rf signals, which are partially picked up by the bottom electrode area as well. Since the measured frequency range goes up to $100 \mathrm{MHz}$ or even

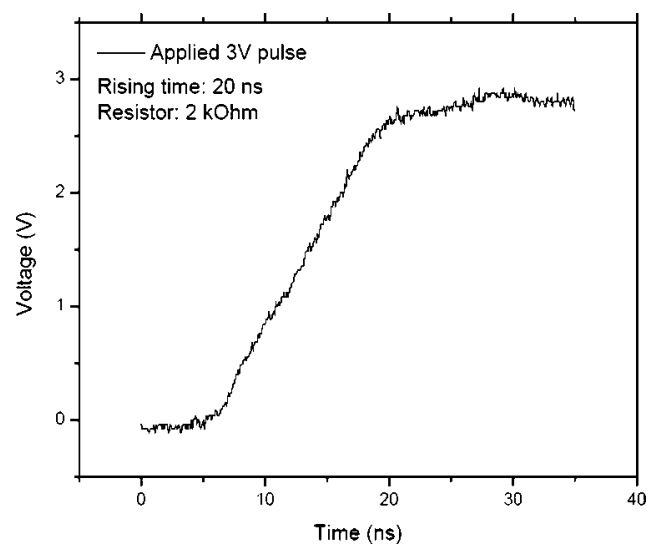

FIG. 3. Calibration voltage pulse used to determine signal delay. 


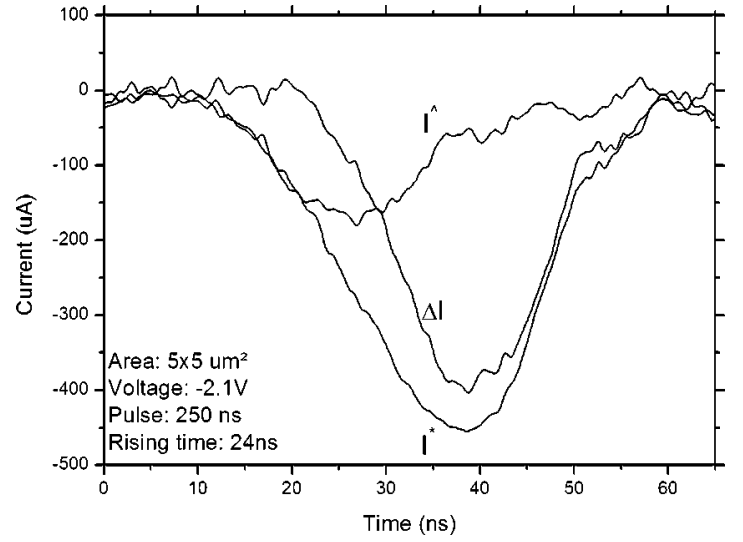

FIG. 4. Transient currents for a $5 \times 5 \mu \mathrm{m}^{2}$ cell and a $-2.1 \mathrm{~V}$ pulses, smoothed via five-point FFT smoothing.

higher, this noise is interlinked into the sensitive measurement circuit. As a result, the minimum transient current that can be measured is in the range of 80 to $100 \mu \mathrm{A}$ without averaging. Using averaging, the minimum measurable current decreases down to $10-20 \mu \mathrm{A}$. The same values are valid for the switching current as well. The maximum loading current of a $3 \times 3 \mu \mathrm{m}^{2}$ cell at $2 \mathrm{~V}$ is in a range of about $150 \mu \mathrm{A}$, which explains the limitation in cell size. The loading current is the lowest current that has to be measured so this current gives the lower limit.

The whole system may be software controlled using LABVIEW in combination with a GPIB bus system. The software allows one to control all the important features of the generator and oscilloscope. Furthermore, it allows processing the (PUND) wave train ${ }^{18}$ in two steps, including the automated calculation of the switching current. Also averaging, different trigger and individual pulse settings for up to two pulse sets are possible. All obtained data may be saved in an ORIGIN compatible file, including parameter settings.

The PUND wave train is a common tool for measuring ferroelectric switching currents. First, the cell must be completely switched to its negative state. Then a positive $P$ pulse is applied. This pulse switches the polarization and is loading the cell. The transient current $\left(I^{*}\right)$ consists of switching current and loading current. The pulse must make sure that the cell was fully loaded and all domains must have switched completely. Then only a nonswitching (loading) current $\left(I^{\wedge}\right)$ is generated by applying a second positive pulse ( $U$ pulse). By subtracting $I^{\wedge}$ from $I^{*}$ the positive switching current $(\Delta I)$
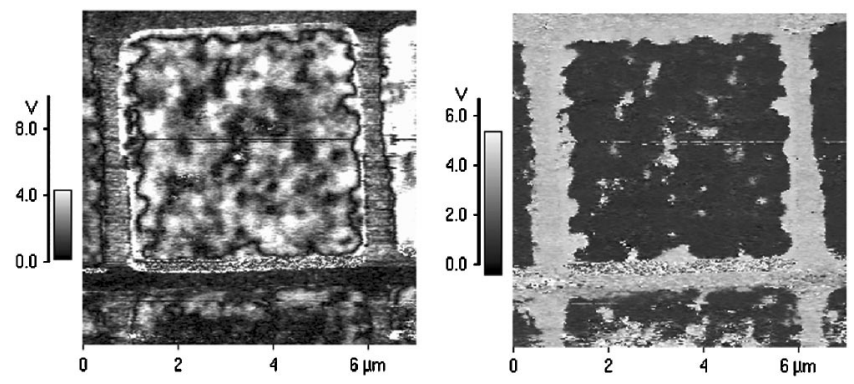

FIG. 5. PFM magnitude (left) and phase (right) images of a $5 \times 5 \mu \mathrm{m}^{2}$ cell switched by a $-2.1 \mathrm{~V}, 250 \mathrm{~ns}$ voltage pulse.
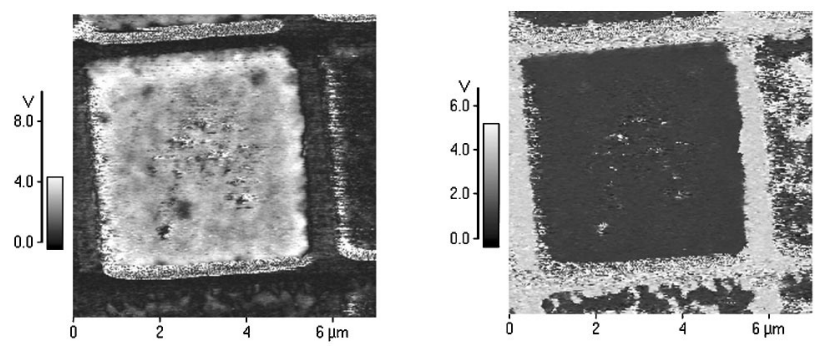

FIG. 6. PFM magnitude (left) and phase (right) images of a $5 \times 5 \mu \mathrm{m}^{2}$ cell switched by a $-5 \mathrm{~V}, 1 \mathrm{~s}$ voltage pulse.

can be found. The same procedure follows for negative switching where the corresponding pulses are called $N$ or $D$ pulses, respectively.

\section{RESULTS AND DISCUSSION}

Figure 4 shows the measurement results for $N\left(I^{*}\right)$ and $D\left(I^{\wedge}\right)$ pulses at a $5 \times 5 \mu \mathrm{m}^{2}$ cell switched by $-2.1 \mathrm{~V}, 250 \mathrm{~ns}$ pulse. All current curves are not averaged but smoothed using a five point fast Fourier transform (FFT) curve smoothing. In Fig. 5 the corresponding PFM image of the cell is shown. It can be seen that while the phase image exhibits almost uniform dark contrast with few bright spots corresponding to remnant domains, the amplitude image presents significant variation of the contrast. For comparison, Fig. 6 shows the same cell after it was switched with the $-5 \mathrm{~V}, 1 \mathrm{~s}$ pulse. Now the cell exhibits uniform contrast both in the phase and amplitude images. It can be concluded that $-2.1 \mathrm{~V}, 250 \mathrm{~ns}$ pulse does not lead to complete polarization reversal leaving nonthrough domains in the capacitor although transient current indicates the end of the switching. ${ }^{19,20}$ Another interesting feature that can be seen from the comparison of Figs. 5 and 6 is slower switching along the capacitor edges, indicating spatial variations in local switching times. Work is underway to investigate the mechanism of this effect. Also, we plan to monitor the backswitching current in order to rule out domain instability during these experiments. In general, the obtained results illustrate the usefulness of combined PFM and current testing of the switching behavior of ferroelectric cells.

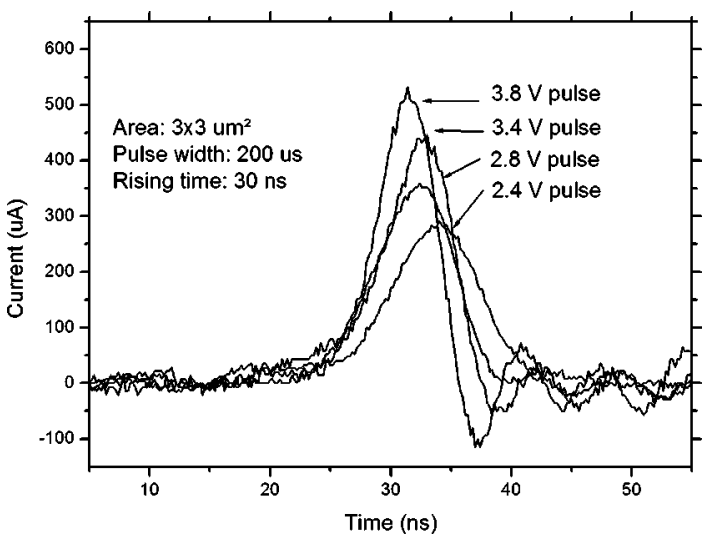

FIG. 7. Switching currents measured a $3 \times 3 \mu \mathrm{m}^{2}$ cell at different voltage pulse amplitudes (pulse duration $200 \mu$ s). 


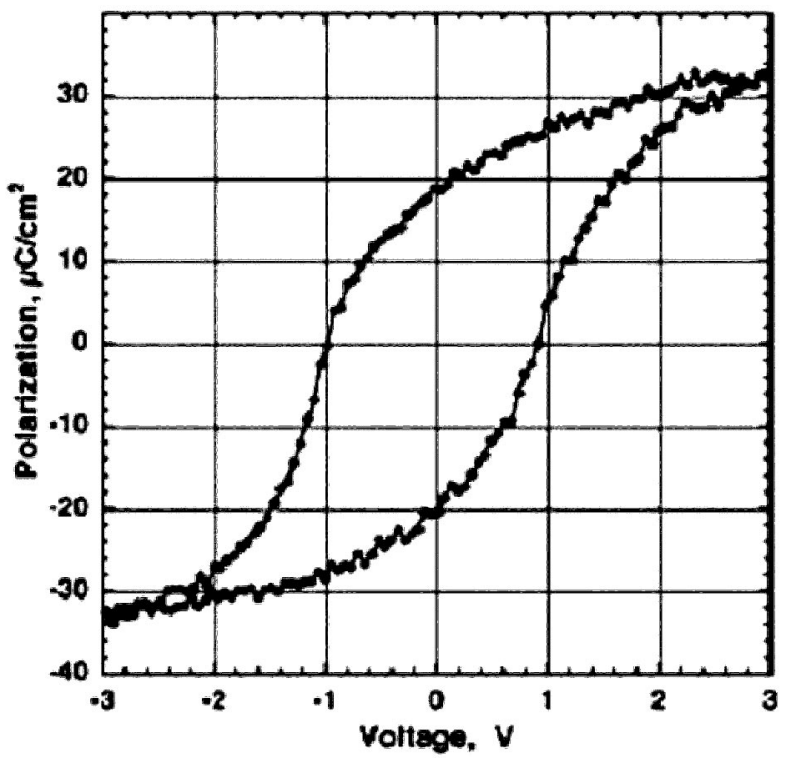

FIG. 8 . Hysteresis loop for a $15 \times 15 \mu \mathrm{m}^{2}$ cell obtained with conventional hysteresis measurement methods.

As mentioned above, our system is able to measure $\Delta I$ of $3 \times 3 \mu \mathrm{m}^{2}$ cells without averaging. We have done measurements at a $3 \times 3 \mu \mathrm{m}^{2}$ cell for eight different voltages between 2.1 and $4.1 \mathrm{~V}$. Figure 7 shows a selection of four of them.

From measured currents the spontaneous polarization can be found as

$$
P_{s}=\frac{Q}{A}=\frac{1}{A} \int i d t
$$

where $i$ is the switching current, $Q$ is the switching charge, and $A$ is the area of top electrode.

The value of the switching charge density in the range between 0.30 and $0.35 \mathrm{C} / \mathrm{m}^{2}$ calculated by integrating the switching current data, is in reasonable agreement with the value of spontaneous polarization obtained via conventional hysteresis loop measurements of the PZT cells (Fig. 8). ${ }^{21}$ The observed difference can be explained by incomplete switching of polarization during pulse measurements as was mentioned above (Fig. 5).

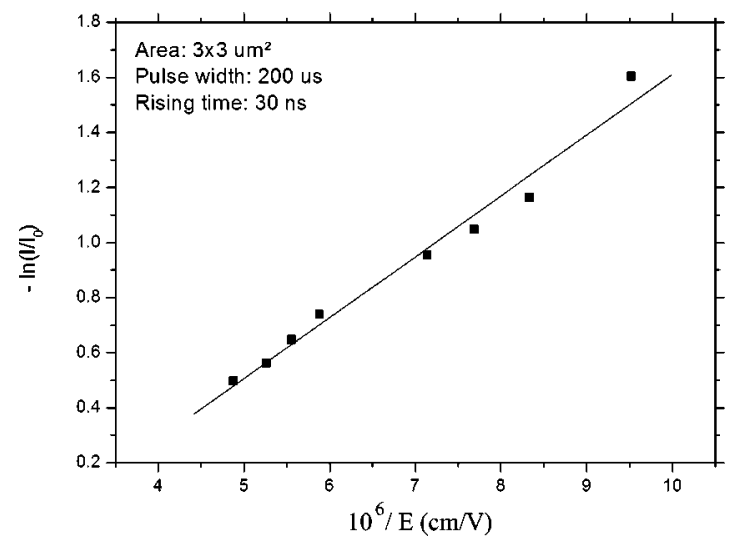

FIG. 9. Switching current vs the applied electric field illustrating the activation type of the switching behavior. Fitting is made using Eq. (2).

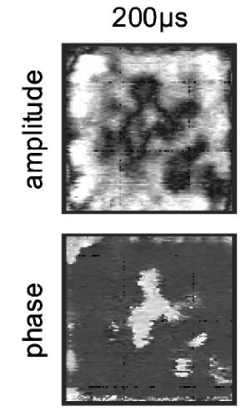

(a)

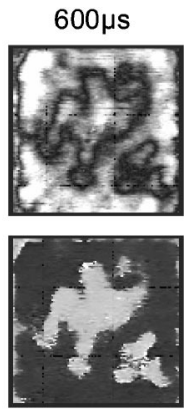

(b)

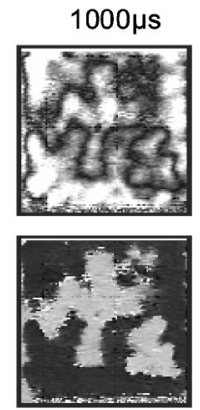

(c)
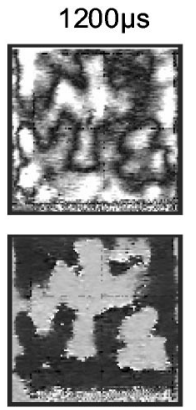

(d)
FIG. 10. PFM magnitude and phase images of instantaneous domain configurations appearing in $3 \times 3 \mu \mathrm{m}^{2}$ FRAM cell during switching by a $1.1 \mathrm{~V}$ pulse at: (a) $200 \mu \mathrm{s}$; (b) $600 \mu \mathrm{s}$; (c) $1000 \mu \mathrm{s}$; and (d) $1200 \mu \mathrm{s}$.

Figure 9 shows the field dependence of maximum switching current $i_{\max }$ obtained from the data in Fig. 7. The dependence can be approximated by the exponential equation found by $\mathrm{Merz}^{22}$ suggesting a well-known activation type switching:

$$
i_{\max }=i_{0} \exp \left(-\frac{E_{a}}{E}\right)
$$

where $i_{0}$ is the experimental constant, $E$ is the applied electric field, and $E_{a}$ is the activation field. From Fig. 9 the activation field for this cell (200-nm thick) was found to be $221 \mathrm{kV} / \mathrm{cm}$, which is consistent with previously reported data. $^{18}$

Limitations of this setup are a long rise time and a high minimum pulse width. The results indicate that the main switching current flows within the first $50 \mathrm{~ns}$. Therefore, using a different pulse generator, capable of achieving $10-15 \mathrm{~ns}$ pulse width and a rise time less than $10 \mathrm{~ns}$, will allow significant improvements in the time resolution. Especially, the rise time will be critical to achieve since problems with ringing will increase due to minimum cable length, given by the setup. Future improvements could be made by installing current measurement equipment close to the AFM head so cables could be shortened.

The developed system can be used to investigate the domain structure dynamics during polarization reversal using the so-called step-by-step switching of FRAM cells. In this approach, partial polarization reversal is induced by applying a voltage pulse that is shorter than the total switching time for the capacitor with subsequent PFM imaging of the resulting domain pattern. By applying a sequence of such short pulses with incrementally increasing duration and by imaging a domain pattern after each pulse a consistent picture of domain dynamics can be obtained. This approach closely emulates the measurement procedure used for testing NVFRAM devices.

Figure 10 shows magnitude and phase images for a 3 $\times 3 \mu^{2}$ cell at different moments of the polarization reversal process. One can easily see the sideways domain growth process during polarization reversal. Detailed studies of domain growth dynamics in PZT thin film capacitors using this setup are under way. 


\section{ACKNOWLEDGMENTS}

The authors are grateful to Dr. I. Stolichnov for discussions related to switching current measurements. This study is supported by the National Science Foundation (Grant No. DMR-0235632) and by the U.S.-Israel Bilateral Science Foundation (BSF) and Fujitsu Limited.

${ }^{1}$ J. F. Scott and C. A. Araujo, Science 246, 1400 (1989).

${ }^{2}$ A. Gruverman, B. J. Rodriguez, R. J. Nemanich, A. I. Kingon, J. S. Cross, and M. Tsukada, Appl. Phys. Lett. 82, 3071 (2003).

${ }^{3}$ S. Tiedke, T. Schmitz, K. Prume, A. Roelofs, T. Schneller, U. Kall, R. Waser, C. Ganpule, V. Nagarajan, A. Stanishefsky, and R. Ramesh, Appl. Phys. Lett. 79, 3678 (2001).

${ }^{4}$ I. Stolichnov, E. Colla, A. Tagantsev, S. S. N. Bharadwaja, S. Hong, N. Setter, J. Cross, and M. Tsukada, Appl. Phys. Lett. 80, 4804 (2002).

${ }^{5}$ J. Li, B. Nagaraj, H. Liang, W. Cao, Chi. H. Lee, and R. Ramesh, Appl. Phys. Lett. 84, 1174 (2004)

${ }^{6}$ S. Prasertchoung, V. Nagarajan, Z. Ma, R. Ramesh, J. S. Cross, and M. Tsukada, Appl. Phys. Lett. 84, 3130 (2004).

${ }^{7}$ A. Gruverman, B. J. Rodriguez, R. J. Nemanich, A. I. Kingon, A. Tagantsev, J. S. Cross, and M. Tsukada, Appl. Phys. Lett. 83, 728 (2003).

${ }^{8}$ O. Auciello, A. Gruverman, and H. Tokumoto, Integr. Ferroelectr. 15, 107 (1997).

${ }^{9}$ M. Alexe, C. Harnagea, D. Hesse, and U. Gosele, Appl. Phys. Lett. 79,
242 (2001).

${ }^{10}$ E. L. Colla, S. Hong, D. V. Taylor, A. K. Tagantsev, and N. Setter, Appl. Phys. Lett. 72, 2763 (1998).

${ }^{11}$ Seungbum Hong, E. L. Colla, Eunah Kim, D. V. Taylor, A. K. Tagantsev, P. Muralt, Kwangsoo No, and N. Setter, J. Appl. Phys. 86, 607 (1999).

${ }^{12}$ A. N. Kolmogorov, Izv. Acad. Nauk USSR., Ser Math. 3, 355 (1937).

${ }^{13}$ V. Y. A. Shur, I. S. Baturin, E. I. Shishkin, and M. V. Belousova, Ferroelectrics 291, 27 (2003)

${ }^{14}$ M. Avrami, J. Chem. Phys. 7, 1103 (1939); 8, 212 (1940); 9, 177 (1941).

${ }^{15}$ J. A. Christman, R. R. Woolcott, Jr., A. I. Kingon, and R. J. Nemanich, Appl. Phys. Lett. 73, 3851 (1998).

${ }^{16}$ A. Gruverman, O. Auciello, and H. Tokumoto, Annu. Rev. Mater. Sci. 28, 101 (1998).

${ }^{17}$ S. Bernacki, L. Jack, Y. Kisler, S. Collins, S. D. Bernstein, R. Hallock, B. Armstrong, J. Shaw, J. Evans, B. Tuttle, B. Hammetter, S. Rogers, B. Nasby, J. Henderson, J. Benedetto, R. Moore, B. Pugh, A. Fennelly, and T. Brown, 4th International Symposium on Integrated Ferroelectrics (ISIF1992), Monterey, CA, March 1992.

${ }^{18}$ D. H. Chang, Y. S. Yoon, and S. J. Kang, J. Korean Phys. Soc. 38, 277 (2001).

${ }^{19}$ I. Stolichnov, A. Tagantsev, and N. Setter, Appl. Phys. Lett. 83, 3362 (2003).

${ }^{20}$ B. J. Rodriguez, A. Gruverman, A. I. Kingon, and R. J. Nemanich, J. Appl. Phys. 95, 1958 (2004).

${ }^{21}$ C. Dehoff (unpublished).

${ }^{22}$ W. J. Merz, Phys. Rev. 95, 690 (1954). 